\title{
Searching Clue of the Relationship between the Alteration of Bioactive Ingredients and the Herbal "Property" Transformation from Raw Rehmanniae Radix (Sheng-Di-Huang) to Steam-Heating-Processed Rehmanniae Radix (Shu-Di-Huang) by Chromatographic Fingerprint Analysis
}

\author{
Peishan Xie ${ }^{1 *}$, Longgang Guo1, Yu Zhao², Daniel Bensky33, Erich Stoeger ${ }^{4}$ \\ ${ }^{1}$ Guangdong UNION Biotechnology Development Co., Ltd., Guangzhou, China \\ ${ }^{2}$ Nikyang Enterprise Co., Ltd., Hong Kong, China \\ ${ }^{3}$ Independent Scholar, Seattle, USA \\ ${ }^{4}$ Plantasia GmbH, Oberndorf, Austria \\ Email: ${ }^{*}$ xps340112@gmail.com
}

Received 20 February 2014; revised 22 March 2014; accepted 9 April 2014

Copyright (C) 2014 by authors and Scientific Research Publishing Inc.

This work is licensed under the Creative Commons Attribution International License (CC BY).

http://creativecommons.org/licenses/by/4.0/

\section{(c) (†) Open Access}

\begin{abstract}
"Di-Huang" (Rehmanniae Radix) is a peculiar Chinese herbal drug, the fresh root named "Xian-DiHuang" ("Xian" means fresh; the commerce is now rarely available), mild-baking-dried raw roots "Sheng-Di-Huang" ("Sheng" means raw) and steam-processed "Shu-Di-Huang" ("Shu" implies steam-processed). The both kinds of herbal drug exert different efficiencies with different property according to the traditional clinical theory. It would be of interest that searching the clue of why the therapeutic functions of the same drug differ between the pre- and post-processing. To disclose the comprehensive alteration of the iridoids, saccharides and phenylethanoids occurred between the raw and steam-processed "Di-Huang", the HPTLC and HPLC chromatographic fingerprint analysis has been carried out. The results showed that in the raw crude drug, the iridoids like catalpol is the major bioactive components, and the oligosaccharides composition kept rather consistent; however, in the steam-processed Di-Huang, iridoids was almost disappeared and the oligosaccharides were hydrolyzed gradually through the repeated steam-heating process. The worst case showed only fructose was detected with other minor residual components in the root

${ }^{*}$ Corresponding author.

How to cite this paper: Xie, P.S., Guo, L.G., Zhao, Y., Bensky, D. and Stoeger, E. (2014) Searching Clue of the Relationship between the Alteration of Bioactive Ingredients and the Herbal "Property" Transformation from Raw Rehmanniae Radix (Sheng-Di-Huang) to Steam-Heating-Processed Rehmanniae Radix (Shu-Di-Huang) by Chromatographic Fingerprint Analysis. Chinese Medicine, 5, 47-60. http://dx.doi.org/10.4236/cm.2014.52006
\end{abstract}


tuber. It is due to presumably the uncontrollable steaming process in folk workshops. Defining reasonably the boundary between the "Sheng-Di-Huang" (mild-baking-dried Rehmanniae radix) and "Shu-Di-Huang" (steaming processed Rehmanniae radix) set up by chromatographic fingerprint pattern and standardizing the process would be necessary for assuring the consistent quality of the commercial herbal drugs.

\section{Keywords} Rehmanniae Radix, Di-Huang, Processing Incurred Herbal-Property-Change, Chromatographic
Fingerprinting, Meaningful Quality Control

\section{Introduction}

Rehmanniae radix is the fresh or dried tuberous roots of Rehmannia glutinosa Libosch, belonging to the family Scrophulariaceae. It has been commonly used in clinics in China for thousands of years. The drug is usually collected in autumn, removed from root stock, rootlet and soil, and used either in fresh state or mild-baked to dryness. The former is known as "Xian Di-Huang" (fresh rehmannia root) which is nowadays rarely available in the market because it is hard to store and transport; the latter is known as "Sheng-Di-Huang" (dried raw Rehmannia root). Since the Tang Dynasty (618 - 907 AD), according to long historical clinical experience, Chinese medicine practitioners considered the property of Sheng-Di-Huang inclined to be used as detoxification for reducing the "heat" in blood, and promoting the production of body fluid and saliva, so its property was relatively "cold". For the patients of "Kidney-Yin"-deficiency, Di-Huang should be transformed to have a "warm" property by repeated steaming process until the crude drug turned black and was called as "Shu-Di-Huang" (steamprocessed Rehmnania radix or prepared Rehmannia radix). This drug is used to nourish "yin" and replenish blood, reinforce the essence of life and marrow and tonify the "Yin-deficient Kidney" [1] [2].

According to the description of "Sheng-Di-Huang" in Chinese Pharmacopoeia [2], the root tuber has brownish-black or brownish-grey external appearance, the texture is heavy, soft and tenacious, fracture surface is brownish black or jet-black, lustrous and viscous. The "Shu-Di-Huang", external is black, lustrous, and stickier. Texture is soft and flexible, uneasily broken, fracture surface is jet-black, lustrous.

The transformation of herbal property from "cold" to "warm" through a steaming process implies that some significant alteration of the inherent secondary metabolites in Di-Huang roots occurs during the processing cycles. The three kinds of the major bioactive ingredients in Di-Huang are iridoids, saccharides and phenylethanols [3]-[7]. For quantitative determination, the current methods are to determine the content of catalpol and rehmannioside D for Sheng-Di-Huang; while acteoside and 5-hydroxymethylfurfural (5-HMF) for Shu-Di-Huang [2] [8]. Some research focused on the contents of selected constituents [8]-[11]. Those isolated markers' determinations are insufficient for searching the clue of the drug's property change. The unfolded global view of two kinds of chromatographic fingerprints of Di-Huang would provide the substantial evidence of dynamic changes of the main bioactive components and possible linkage to the drug properties transformation. The information will serve as the fundamental background for establishing a reliable specification to ensure the meaningful quality control standard.

In this study, the associated fingerprints of High-performance Thin-layer Chromatography (HPTLC) and High-performance Liquid Chromatography (HPLC) complementarily represented the characteristics of ShengDi-Huang and Shu-Di-Huang respectively. According to the fingerprint patterns, the relativity between the dynamic changes of the chemical composition and the drug property transformation from Sheng-Di-Huang to ShuDi-Huang is discussed in this paper.

\section{Experimental}

\subsection{Sample and Apparatus}

- Samples

39 batches of Rhmannia root (root of Rehmannia glutinosa L.; Di-Huang) were utilized, 17 batches from He- 
nan province, the rests purchased from herbal drug markets in the provinces of Guangdong, Guizhou, Jiangxi, Fujian, Hunan, Anhui, Hebei and Guangxi.

- Chemicals and reagents

Catalptol and acetoside (purchased from Chinese National Institute for the Control of Pharmaceutical and Biological products, Beijing, China); rehmannioside D (provided by State Administration of Traditional Chinese herbal medicine of People's Republic of China, Beijing, China); raffinose (purchased from Sinopharm Chemical Shanghai Chemical Reagent Co. Ltd., Shanghai, China); sucrose (purchased from Tianjin Guangfu Fine Chemical Research Institute, Tianjin, China); D-fructose (Purchased from Sinopharm Chemical Reagent Co. Ltd., Shanghai, China); Stachyose (Purchased from Xi-An Dapeng Biological Technology Co. Ltd., Xi'an, China).

All chemicals and reagents are of analytical grade.

- Apparatus

HPTLC were performed on High performance silica gel pre-coated plate (Merck, Germany), Automatic TLC sampler 4, TLC development twin-trough chamber, TLC scanner 3 with WinCats software, Digistore TLC documentation device and software (all from CAMAG, Switzerland); TLC digiscan-Acquiring software (developed by Franky Du of Nikyang Enterprises Company, Hong Kong).

HPLC were performed on an Agilent 1100 series HPLC system with autosampler and diode array detector (DAD).

HPLC column: ZORBAX SB C18 $(4.6 \times 250 \mathrm{~mm}, 5 \mu \mathrm{m}$, Agilent).

\subsection{Preparation of Sample Solution}

- Sample solution for HPTLC analysis

$0.1 \mathrm{~g}$ of powdered sample were placed in a graduated test tube, add $5 \mathrm{~mL}$ of methanol, ultrosonicate at $40^{\circ} \mathrm{C}$ for 15 minutes, centrifuge for 10 minutes ( $4000 \mathrm{r} / \mathrm{min}$ ), use the supernatant as sample solution.

- Sample solution for HPLC analysis

Add $50 \mathrm{~mL}$ of $70 \%$ ethanol to $2 \mathrm{~g}$ of powdered sample, reflux on a $90^{\circ} \mathrm{C}$ water bath for 30 minutes, filter, evaporate the filtrate to dryness on a $90^{\circ} \mathrm{C}$ water bath, dissolve the residue in $20 \mathrm{~mL}$ of water and transfer to a separating funnel, extract with $30 \mathrm{~mL}$ of n-butanol twice, combine the n-butanol, evaporate to dryness on a $90^{\circ} \mathrm{C}$ water bath, dissolve the residue in $30 \%$ ethanol and transfer to a $5 \mathrm{~mL}$ of volumetric flask and dilute to volume, mixed well, then filter through $0.45 \mu \mathrm{m}$ filter membrane, use the final filtrate as sample solution.

\subsection{Preparation of Chemical Reference Solution}

Dissolve $5 \mathrm{mg}$ of the Chemical Reference Substances (CRS) cataltol and $8 \mathrm{mg}$ of rehmannioside D in $1 \mathrm{~mL}$ methanol, respectively. Dissolve $2 \mathrm{mg}$ of raffinose, $2 \mathrm{mg}$ of sucrose and $0.5 \mathrm{mg}$ of D-fructose chemical reagent in 1 $\mathrm{mL}$ of $70 \%$ ethanol, respectively. Use these as saccharides and iridoids reference solution for HPTLC analysis.

Accurately weight a quantity of acteoside (CRS), dissolve in 30\% methanol to produce a solution containing $0.5 \mathrm{mg}$ per $1 \mathrm{ml}$ as reference solution for HPLC analysis.

\subsection{Experiment Procedure}

- HPTLC fingerprint of Rehmannia root (Di-Huang) collected from different places Stationary phase: HPTLC plate $(20 \times 10 \mathrm{~cm}$; Merck; batch Number: HX604153).

Mobile phase: Dichloromethane-ethyl acetate-methanol-water-glacial acetic acid (8:4:7:1:1).

Saturation: Pre-equilibrate the development chamber with the mobile phase for 15 minutes prior to analysis (add about $8 \mathrm{~mL}$ of the solvent mixture into one trough of the chamber).

Sample application: apply $2 \mu \mathrm{L}$ of sample solution and $1 \mu \mathrm{L}$ of chemical reference solution as band onto HPTLC plate respectively. The original application position was $8 \mathrm{~mm}$ above from the bottom edge.

Development: The plates loaded with sample solution were dried in a vacuum dessicator over phosphorous pentoxide for 2 - 3 hours prior to development. The sufficient dried sample-loaded plate was placed promptly in the chamber, developing distance of the mobile phase reached to $8 \mathrm{~cm}$. The residual solvent on the developed plate was dried with a stream of cold air for 10 minutes. Spray with $2 \%$ anisaldehyde in $5 \%$ sulphuric acid ethanol solution, heat at $105^{\circ} \mathrm{C}$ until the bands are clearly visible.

Detection: Observe the fluorescence image under UV light at $366 \mathrm{~nm}$ and documentation was done as usual 
(Figure 1).

- HPLC fingerprint of rehmannia root collected from different place

Column: ZORBAX SB C $18(4.6 \times 250 \mathrm{~mm}, 5 \mu \mathrm{m}$; Aglient $)$

Column temperature: $30^{\circ} \mathrm{C}$

Gradient of mobile phase: A-acetonitrile; B-0.1\% acetic acid in water.

\begin{tabular}{ccc}
\hline Time(min) & A (\%) & B (\%) \\
\hline 0 & 10 & 90 \\
15 & 17 & 83 \\
25 & 17 & 83 \\
55 & 30 & 70 \\
\hline
\end{tabular}

Flow rate: $1 \mathrm{~mL} / \mathrm{min}$

Injection volume: $20 \mu \mathrm{L}$

Detection wavelength: $334 \mathrm{~nm}$ (for phenylethanol glycosides)

Run time: 55 minutes

Compare the chromatogram of sample solution against the chromatogram of reference solution.

\section{Results and Discussion}

\subsection{HPTLC Fingerprint Analysis}

- Characteristics HPTLC fingerprint

The HPTLC fluorescence image of raw dried Rhamannia roots (Sheng-Di-Huang) developed four yellow fluorescent bands of saccharides: stachyose $\left(R_{f}\right.$ value ac. 0.06$)$, raffinose $\left(R_{f}\right.$ value ac. 0.14$)$, sucrose $\left(R_{f}\right.$ value ac. 0.26), D-fructose $\left(R_{f}\right.$ value ac. 0.35$)$ and two grayish-blue fluorescent bands of iridoids (rehmannioside $D\left(R_{f}\right.$ value ac.0.18), catalpol ( $R_{f}$ value ac.0.52). The relative fluorescence abundance of the components in the image can be compared visually (Table 1 ).

The digital scanning profiles of the HPTLC images (Figure 1) expressed more clearly the intact view of the fingerprint of Sheng-Di-Huang. It can serve as the common pattern of the raw material (Figure 2). As a typical Sheng-Di-Huang, among saccharides, strachyose is the strongest fluorescence band, raffinose and sucrose gave stronger or weaker fluorescence bands, fructose is the weakest in different samples; As iridoids, catalpol were obviously detected as grey-blue fluorescence bands, but the content (fluorescence abundance) fluctuated widely

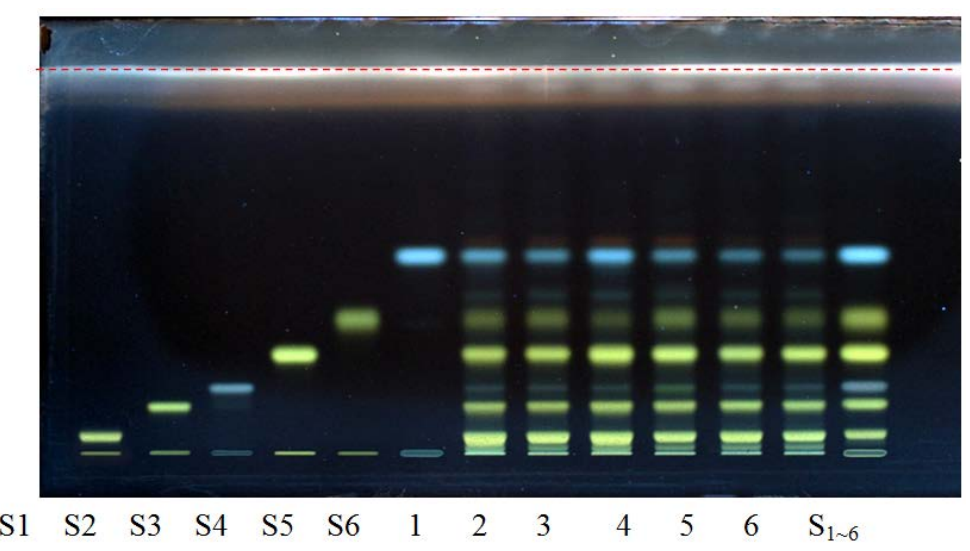

Figure 1. HPTL Cimage of Di-Huang (Rehmanniae Radix). (S1)-Stachyose; (S2)-Raffinose; (S3)-Rehmannioside; (S4)-Surcose; (S5)-D-Fructose; (S6)Catalpol; (1). Reference crude drug; (2)-(6) Commercial samples; $\left(\mathrm{S}_{1 \sim 6}\right)$ mixed chemical reference substances. 


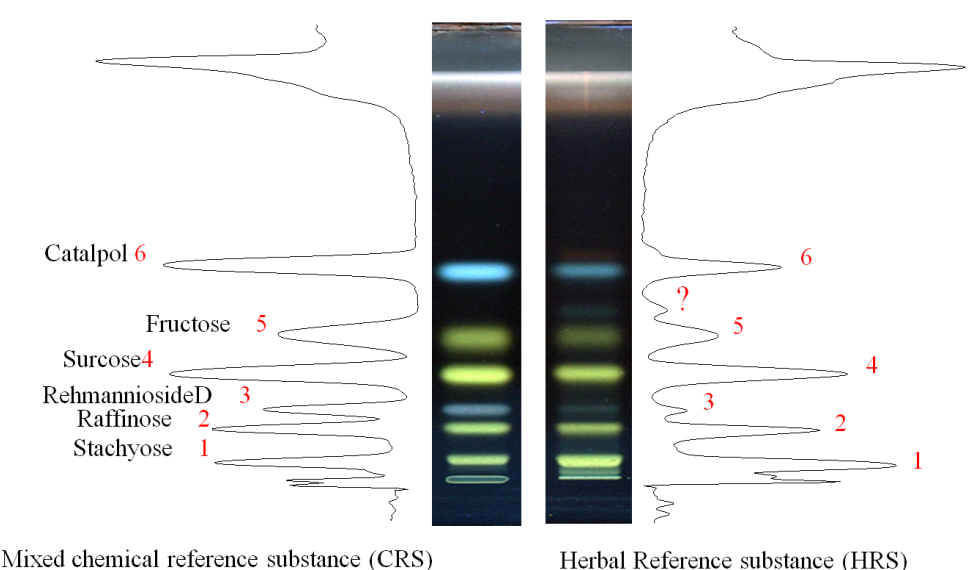

Figure 2. HPTLC images and digital scanning profiles of mixed CRS and HRS of Rehmannia root. Peaks: (1) stachyose; (2) raffinose; (3) rhemannioside D; (4) sucrose; (5) fructose; (6) catalpol, (?) unknown yetsaccharide.

Table 1. Approximate comparison of the fluorescence bands abundance of the saccharides and iridoids in the HPTLC image of Sheng-Di-Huang and Shu-Di-Huang.

\begin{tabular}{ccccccc}
\hline Fluorescent band & $\begin{array}{c}\text { Stachyose } \\
\text { (Rf ca. 0.06) }\end{array}$ & $\begin{array}{c}\text { Raffinose } \\
\text { (Rf ca. 0.14) }\end{array}$ & $\begin{array}{c}\text { Rehmannioside D } \\
\text { (Rf ca.0.18) }\end{array}$ & $\begin{array}{c}\text { Sucrose } \\
\text { (Rf ca. 0.26) }\end{array}$ & $\begin{array}{c}\text { D-fructcoseA } \\
\text { (Rf ca. 0.35) }\end{array}$ & $\begin{array}{c}\text { Catalpol } \\
\text { (Rf ca. 0.52) }\end{array}$ \\
\hline Sheng-Di-Huang & +++ & ++ & + & $++/+++$ & $+/++$ & $+/++/+++$ \\
Shu-Di-Huang & $++/+$ & minor/- & $+/$ trace & $+++/++$ & $++/+++$ & minor/- \\
\hline
\end{tabular}

^ : glucose overlapped with fructose.

in different commercial samples (The literature reported catalpol is the highest content among the iridoids glycosides [11]). Briefly, the intact image and the scanning profile (number of bands, location and order, fluorescence/visible color, strength of the fluorescence abundance, peak height/area ratio between bands) constructed the unique fingerprint pattern of Sheng-Di-Huang.The digital scanning profiles of the HPTLC images (Figure 1) provided more clear intact view of the fingerprint. It can serve as the common pattern of Sheng-Di-Huang (Figure 2).

With regard to the HPTLC image pattern of Shu-Di-Huang, comparison with of Sheng-Di-Huang, the prominent feature is iridoids represented by catalpol were decomposed seriously, even radically disappeared. Secondly, the oligosaccharides were hydrolyzed at different degrees, consequently sucrose, even fructose increased accordingly (Table 1). Therefore the HTPLC image of Shu-Di-Huang showed a rather different visage from ShengDi-Huang. Glucose almost dehydrated to 5-hydrxoy-methyl furfural (5-HMF) (vide infra).

- Dynamic alteration of the active ingredients of the root tuber of Rehmannia subjected to the steaming process

For the sake of investigation of the dynamic alteration of the compounds in Di-Huang, a quantity of "ShengDi-Huang" was steamed in a small bamboo steamer placed in a wok filled with boiling water, heated for 0, 2, 4 and 8 hours; and then a given quantity was taken out as samples, dried under reduced pressure at $60^{\circ}$, powdered, then prepared the sample solutions as described in section 2.2. for HPTLC analysis. The experiment demonstrated the steam-heating tolerance test of oligosaccharides and catalpol. The results indicated that stachyose, raffinose and sucrose in the roots were at rather stable till 4 hours. After that, those all gradually hydrolyzed drastically in different extent, and the D-fructose proportional increased, but stachyose was relatively rather stable against steam-heating (Figure 2). It means that the majority of the oligosaccharides were hydrolyzed to monosaccharide-D-fructose and glucose, only stachyose still exist. Catalpol—-the main iridoid glycoside seriously decreased, even radically disappeared (Figure 3).

On the other hand, the chain-structured monosaccharide like glucose would be decomposed to be 5-hydroxymethylfurfural (5-HMF). The increase trend of 5-HMF along with the steaming period was occurred simultaneously (Figure 4).

- Application of fingerprint to the commercial samples analysis

Generally the commercial samples of Sheng Di-Huang and Shu-Di-Huang collected from various markets, the 


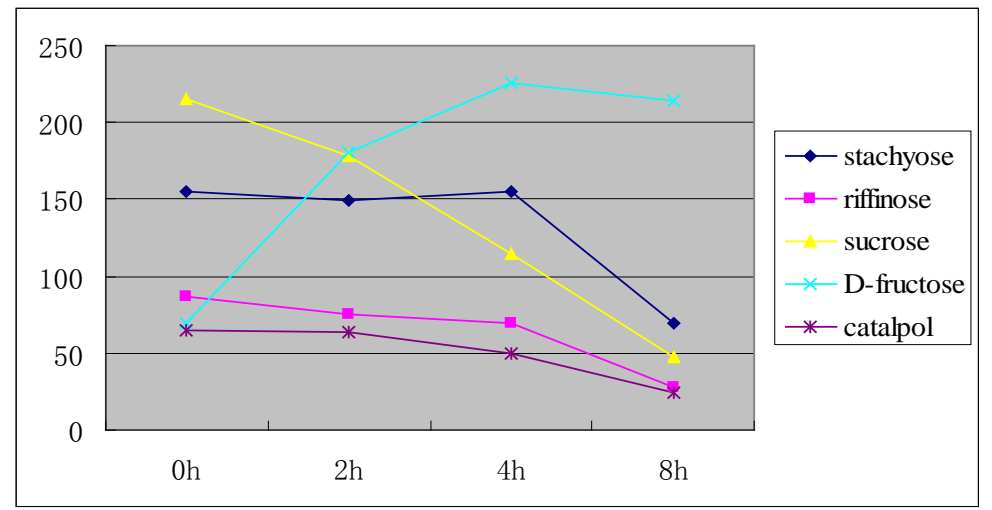

Figure 3. Comparison of peak area of several active components of Di-Huang (Rehmanniae Radix) steamed in persistently prolonged heating.

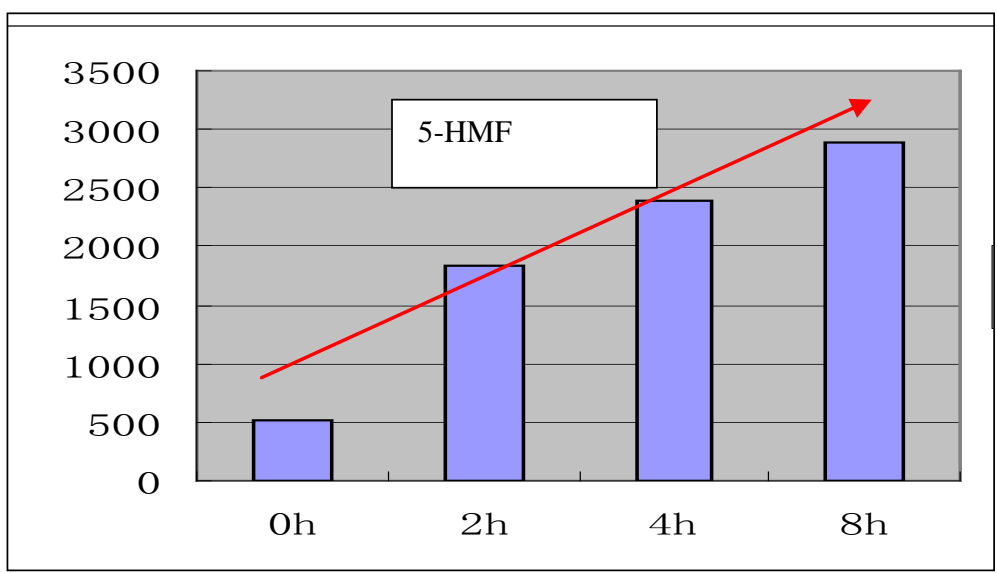

Figure 4. Peak area of 5-hydroxy-methylfurfural (5-MHF) inDi-Huang which was generated from decomposition of glucose when steamed in persistently prolonged heating.

labeled names rely solely on the claims of the suppliers. From the chromatographic fingerprints it is apparent that the ingredients in various samples exhibited some fluctuation which caused by variations in determining methods, sample habitats and uncontrolled steam-heating process [12] [13].

Based on the extent of decomposition of the ingredients, there are four grades of the patterns in the collected commercial samples as shown in the HPTLC fingerprints (images combined with the corresponding scanning profiles) (Figure 5). It is noteworthy that the most important tetra-oligosaccharide—stachyose [14] seems relatively more stable than the other oligosaccharides (Figure 5 and Figure 6).

The project plot of Principle Component Analysis (PCA) of "Di-Huang" disclosed that while some of the commercial samples of "Sheng-Di-Huang" were closely distributed around the common pattern, (Grade I), others spread a distance from the common pattern, located at the upper part (Grade II) which have the typical character of of "Sheng-Di-Huang" common pattern, but catalpol was hard to be detected. Grade III and Grade IV those in the right side belonging to "Shu-Du-Huang". An exceptional example of Shu-Di-Huang (21\#) showed only fructose. (The intact root tuber is of "as black as Chinese lacquer" (e.g., \#21 sample) and always being considered as superior entity in the description of ancient herbal compendia). Obviously in fact such sample suffered from over-steaming (Figures 5-7).

\subsection{HPLC Fingerprint Analysis}

- HPLC fingerprint of Di-Huang

For analysis of the phenylethanol derivatives in Di-Huang, the HPLC-DAD experiment was carried out, the 

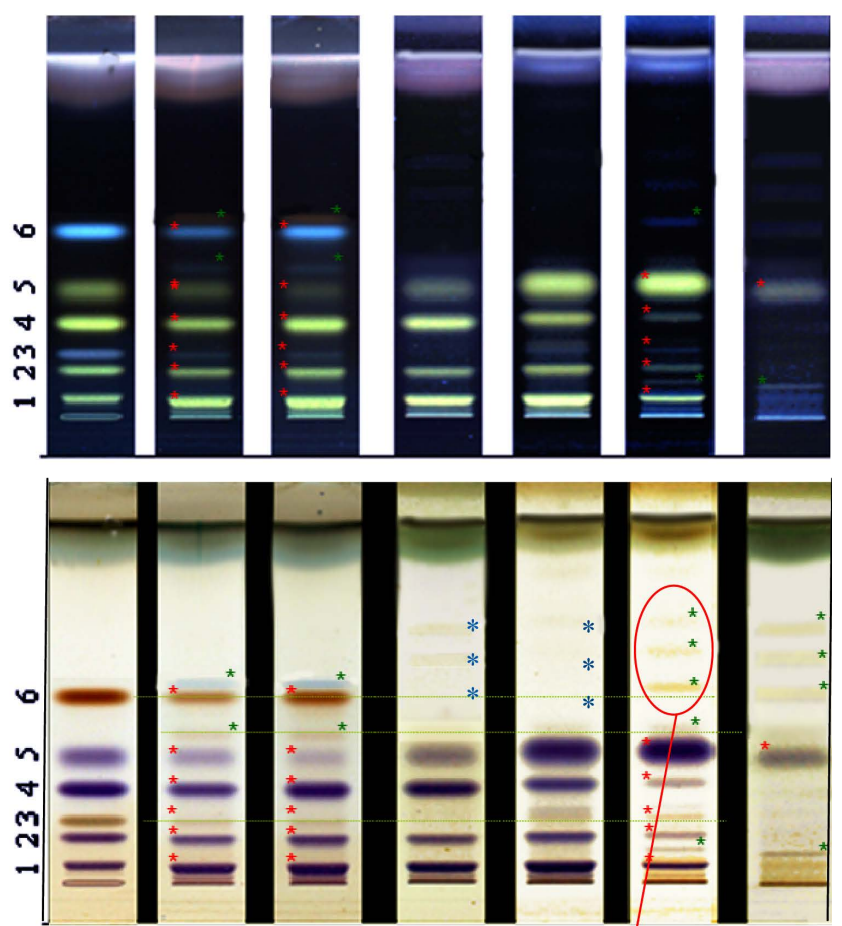

(1)

(2)

(3)

(4)

(5)

(6)

(7)

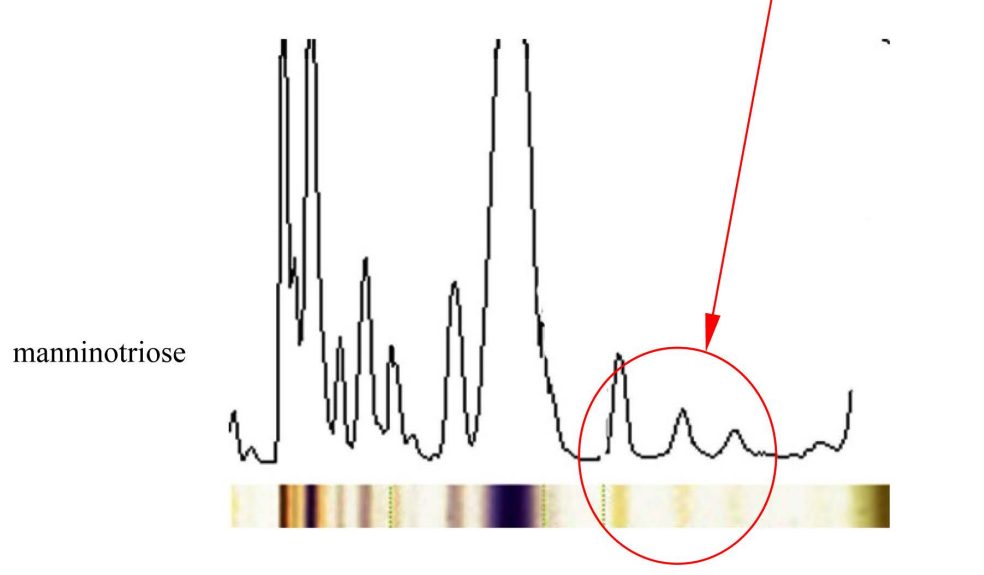

Figure 5. The HPTLC images of "Di-Huang" are divided four grades. (The figure below is the inverted color image from the upper fluorescence figure for more easy comparison), Track (1) Chemical reference substances: (1) stachyose, (2) raffinose, (3) rehmannioside D, (4) sucrose, (5) fructose, (6) catalpol Track (2) authentic sample of "Sheng-Di-Huang"; Track (3) commercial sample of "Sheng-Di-Huang" Track (4) similar appearance of the crude drug with the authentic sample (2) pattern, but catalpol exceptionally disappeared and the rehmanioside D was very weak. (cf. Figure 7). Track (5)-(6) the crude drugs appearances and the HPTLC pattern of the commercial samples were up to the description of "Shu-Di-Huang" in Chinese Pharmacopoeia, but the extent of hydrolysis of the saccharides differ, the monosaccharide-fructose increased drastically. Note that the stachyose (band 1) was relatively more stable than the others. Track (7) an over-steamingprocessed sample of "Shu-Di-Huang", almost all the major ingredients disappeared or only residual amounts remained, but fructose being abundant. The blue stars marked in the invert color images (the figure below) showed some weak chemicals which are almost hard to be recognized under deep color background in the original fluorescence image. 


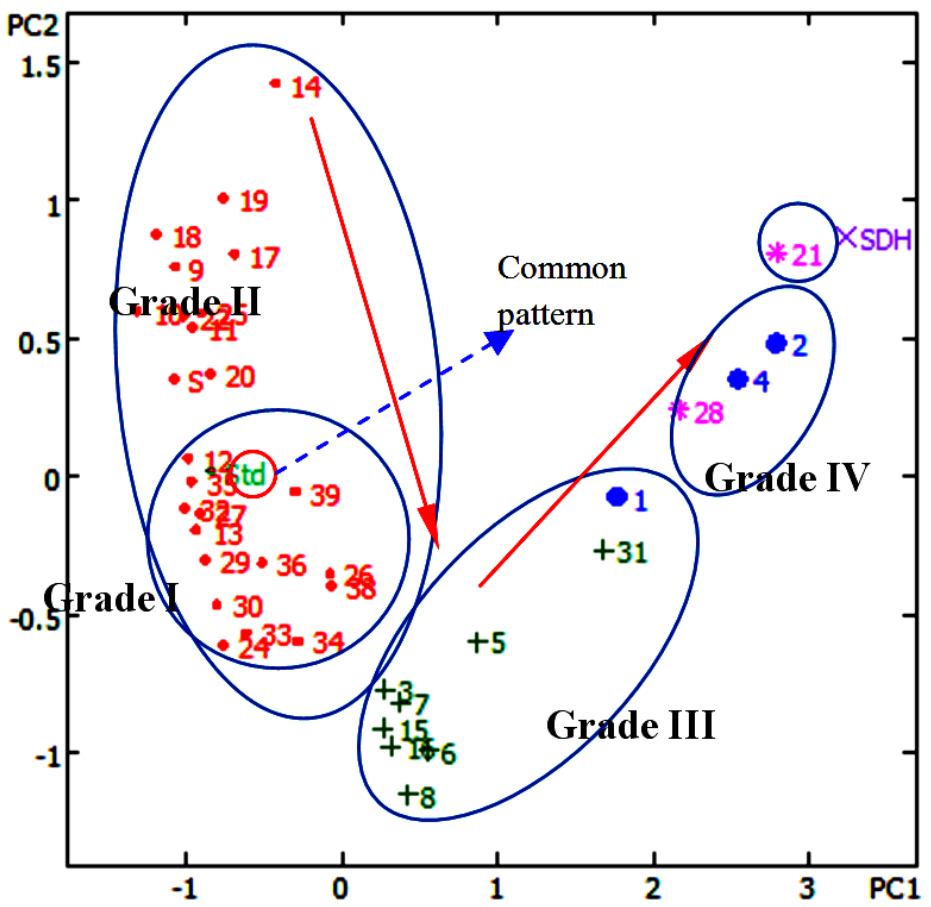

Figure 6. PCA projection plot of HPTLC fingerprint of Di-Huang. The Grade I and Grade II belong to "Sheng-Di-Huang", but the samples in Grade II, catapol was hard to be detected. Grade III and IV expressed the feature of "Shu-Di-Huang", but the samples in Grade IV contain minor amount of catalpol. The dynamic change of the oligosaccharides accompanying the steam processing caused some samples ("Shu-Di-Huang”) distance from the common pattern. The longer the steam-heating time, the more severe the hydrolysis of raffinose and sucrose transforming them into fructose and glucose will occur. Stachyose was rather stable than the other oligosaccharides. (cf. Figure 5). Sample \#21(“SDH”) showed only fructose (+ glucose) dominated in the fingerprint due to over-steam-heating led almost all oligosaccharides hydrolyzed to monosaccharide.

detection wavelength set at UV $334 \mathrm{~nm}$ to meet the maximum absorbance of this kind of components. The HPLC common pattern of "Sheng-Di-Huang" consisted of 11 characteristic peaks including acteoside and other phenylethanols (Figure 8).

- Applied Fingerprint to commercial sample analysis

Similarity of commercial samples has been calculated based on correlation coefficient for comparison with the common pattern (CP) (see Figure 9).

Since the concentration, distribution and proportion between the active components differ from reference crude drug, some samples of relative lower similarity (below 0.85) compared with the common pattern. Samples q\#14 and \#30 are two examples with unusual profiles individually. The supernormal strong peak of acetoside in HPLC profile of sample \#14 also led to lower similarity (correlative coefficient 0.83 ) and oppositely a severe decline peak of acetoside as well as the peak (3) and (4) in the HPLC profile of sample \#30 caused the lowest similarity of 0.24 (Figure 10 and Figure 11).

- Comparison of HPLC fingerprints between Sheng-Di-Huang and Shu-Di-Huang

In HPLC fingerprint of the phenylethanols derivatives of Shu-Di-Huang are also decomposed in some extent by comparison with "Sheng Di-Huang", and increased some of the higher polarity compounds in the region of ca. Rt 4 min to 14 min (Figure 12).

\section{Discussion}

Combining the HPTLC fingerprint (fluorescence images plus the corresponding digital scanning profiles) and 

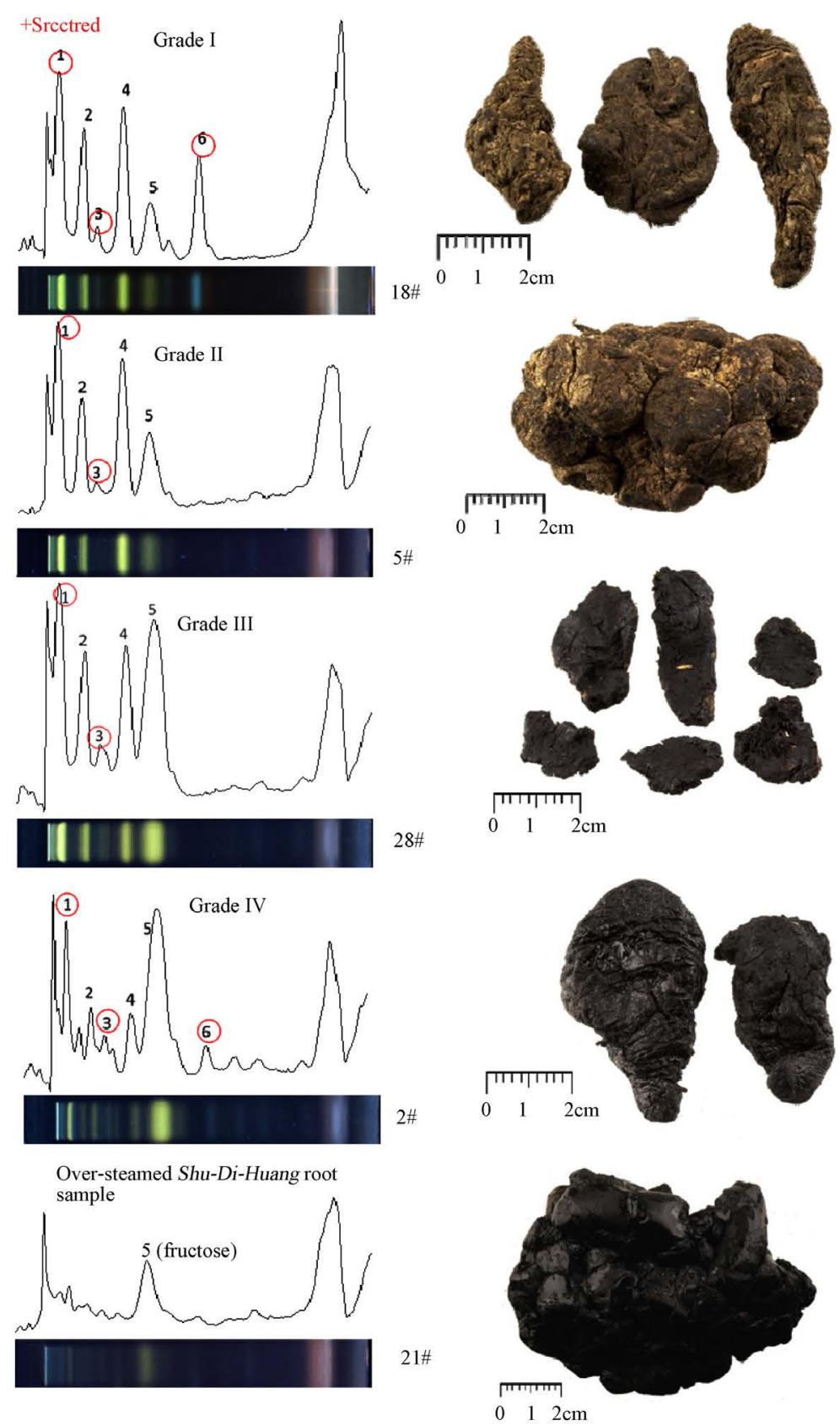

Figure 7. The changes of the HPTLC fingerprints of various commercial DiHuang sample. (1)-Stachyose; (2)-Raffinose; (3)-Rehmannioside D; (4)-Surcose; (5)-D-Fructose; (6)-Catalpol.

the HPLC-DAD fingerprints of "Di-Huang" constructs an integrative view on the characteristics distributions of ingredients in "Sheng-Di-Huang" and "Shu-Di-Huang". The HPTLC fingerprint showed a whole range of detectable iridoids and saccharides. HPLC gave the profile of phenylethanol derivatives. The fingerprints provided useful information to distinguish Di-Huang by means of dual chromatographic analysis. It was revealed that all the bioactive ingredients in Di-Huang are steam-heating-labile. The fingerprint of Sheng-Di-Huang consists of catalpol, rehmannioside D, stachyose, raffinose, sucrose, and fructose as well as some minor unknown components. But those chemical ingredients led to decompose during the steaming process. The loss of catapol and other iridoids and varying degrees of decomposition of saccharides exhibited in the fingerprints of Shu-DiHuang. The dynamic change of the saccharides in HPTLC fingerprint can possibly be traced to the extent of the 


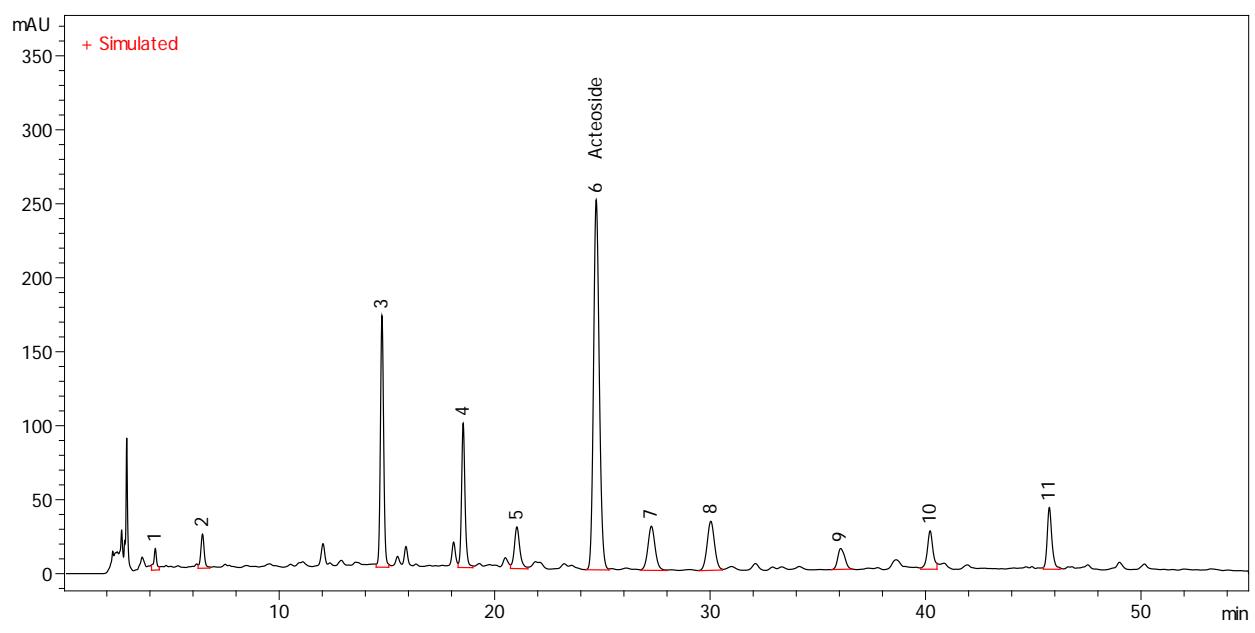

Figure 8. HPLC fingerprint common pattern of dried raw Rehmmaniae radix (Sheng-Di-Huang).

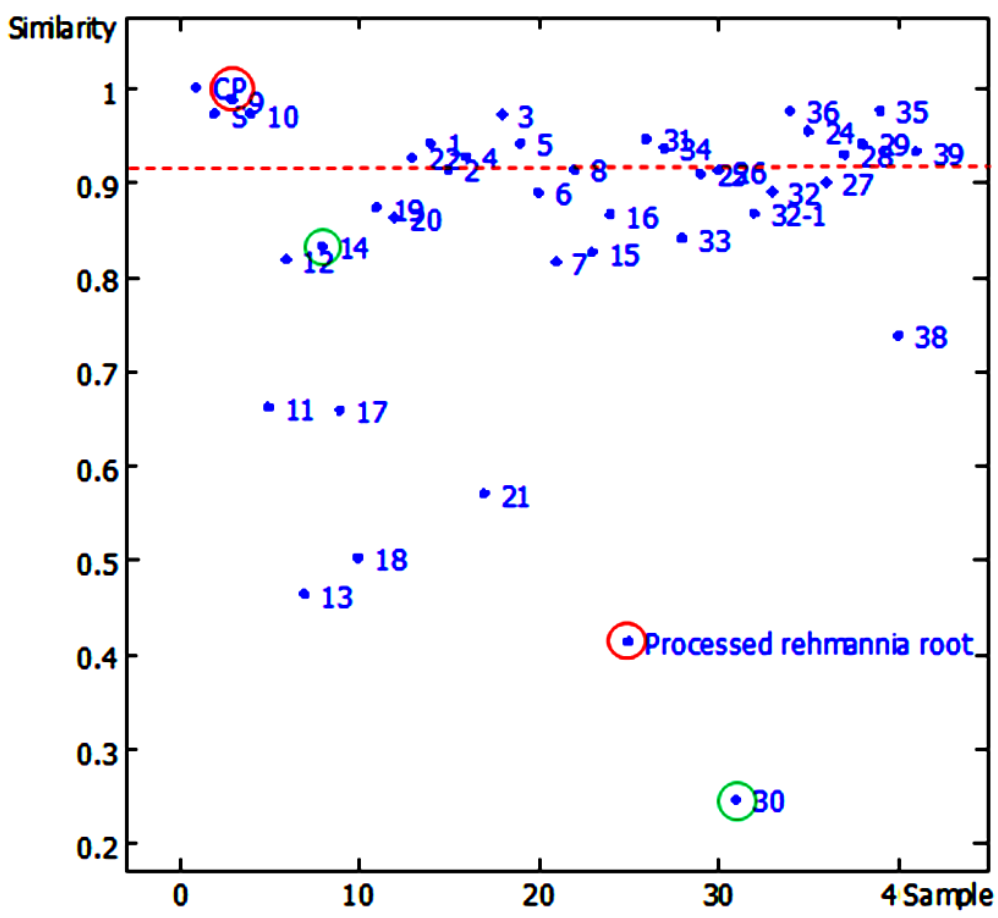

Figure 9. Similarity results of HPLC fingerprint of all samples of Di-Huang.

steaming process. It is noteworthy that stachyose seemed rather stable than the other oligosaccharides as expressed in the fluorescence images of various samples of "Shu-Di-Huang".

Referring to phenylethanol derivatives, acteoside which dominated in the HPLC fingerprint of "Sheng-DiHuang" also decline gradually in Shu-Di-Huang as the steaming process went on.

In a word, "Shu-Di-Huang" cannot preserve the intact appearance of the fingerprint like that of Sheng-DiHuang, either HPTLC (iridoids and oligosaccharides) or HPLC (phenylethanol derivatives) due to instability of the said bioactive metabolites. On the other hand, an ensuing rising of 5-HMF derived from glucose was also a complementary feature of Shu-Di-Huang (Figure 4). Recent studies claimed that 5-HMF is likely also an important bioactive component which exists in many herbal drugs with the function of antioxidant and nourishing kidney Yin deficiency [15]. So its influence to the drug property is also worth considering.

As iridoids and hydrolysable oligosaccharides are unstable, it brought up the possibility of developing a line 

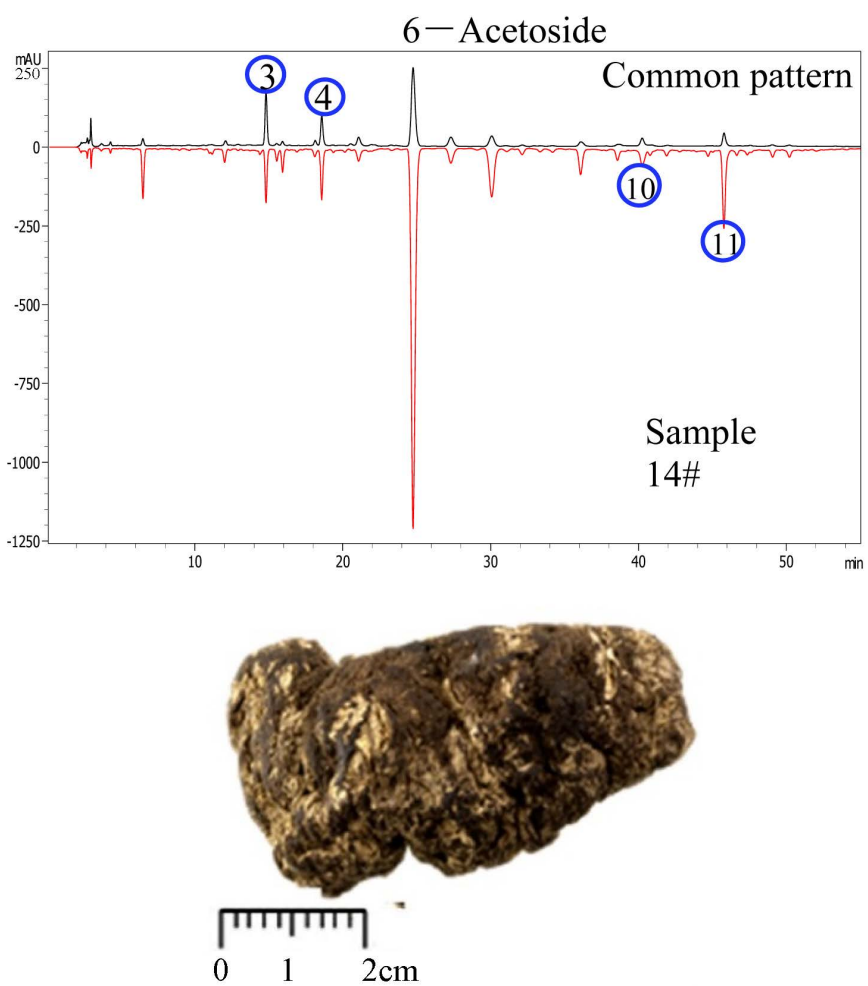

Figure 10. Comparison of HPLC profiles of commercial sample 14\# with common pattern of "Sheng-Di-Huang". the content of acteoside is much higher than that in the common pattern.
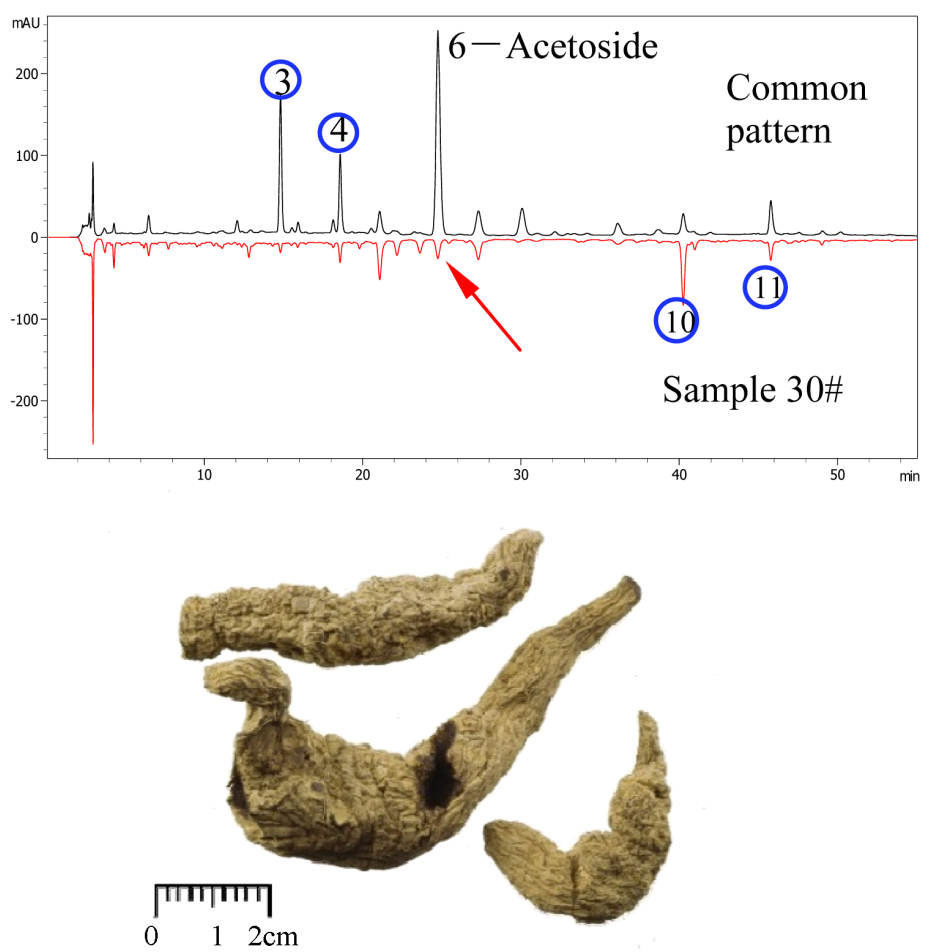

Figure 11. Comparison of HPLC profiles of sample 30\# and the common pattern. The acteoside and other phenylethanol compounds severely declined in sample 30\#,the appearance of which looked as Sheng-Di-Huang. 


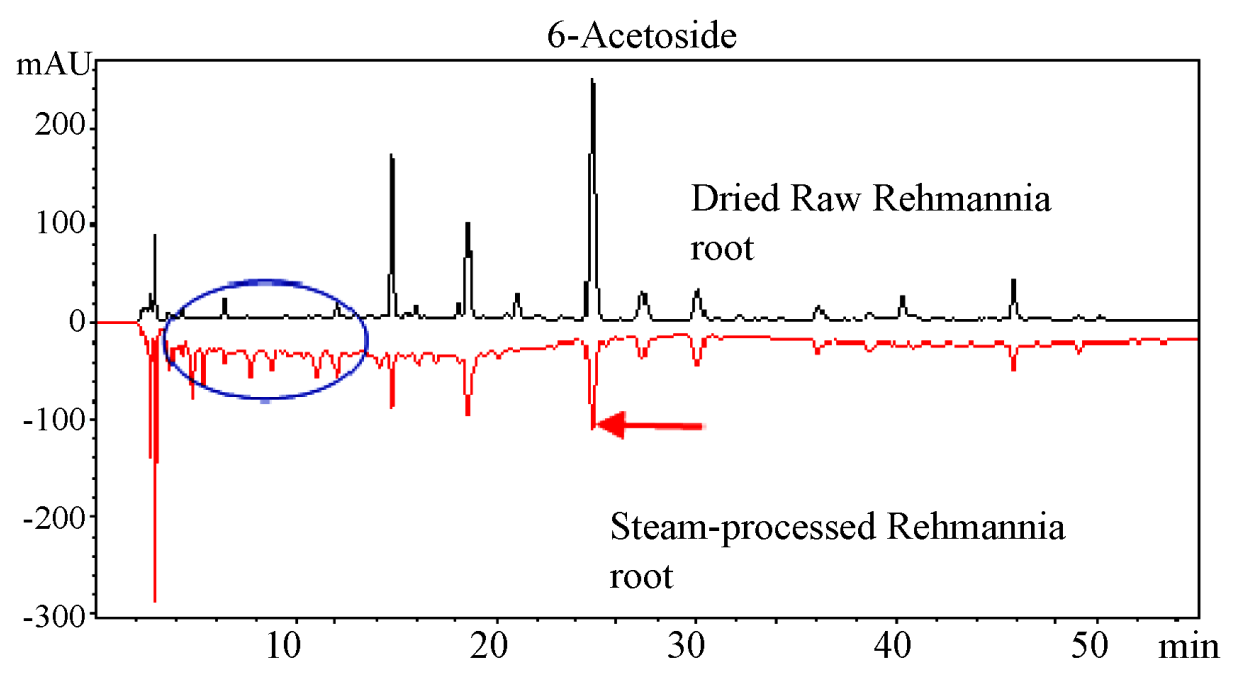

Figure 12. Comparison of HPLC profiles of Sheng-Di-Huang and Shu-Di-Huang.

to divide Di-Huang from "Sheng-" (raw, dried) to "Shu-" (processed, steam-heated). The dynamic alteration of the bioactive ingredients provided some clue on the correlation with the herbal drug's "property" transformation. It might presume from the fingerprint that the iridoids (catalpol is the highest content [8]), in particular, with "cold” property in Sheng-Di-Huang to exert the principle role for "cooling the hot of the blood”, etc.; oligosaccharides, fairly stable stachyose with "warmer" property in Shu-Di-Huang to exert the function of nourishing "yin" of the "kidney", etc. It cannot certainly be neglected that the holistic role with the other chemical components, i.e., phenylethanol glycosides, polysaccharides, etc.; in addition, the 5-HMF derived from dehydration of glucose and fructose during processing has also its contribution to the property transformation in Shu-Di-Huang. But the obvious different distributions of the detectable bioactive constituents in the two kinds of Di-Huang demonstrated in the chemical fingerprints are inferable that the properties of the both Di-Huang have their own individual focus and cannot be considered equivalent based on bioactivity. Modern pharmacological studies have also disclosed the various bioactive functions of different constituents in Di-Huang [15] [21]. The analysis result confirmed that too long steam-heating for getting the root tuber black as lacquer recorded in the historic herbal books cannot be considered the effective bioactive ingredients composition retained. Hence, standardizing the process procedure is necessary. Supposedly, it would be feasible to steady the quality of Shu-Di-Huang with the measures like cutting the root tuber into small pieces, improving the facilities, fix the time of steaming, enhancing the in-process quality control. Further in-depth investigation on the causal relationship between the chemical fingerprints and the traditional recorded Chinese medicine uses in a holistic way can be expected in the future.

\section{Conclusion}

The inconsistent chemical profiles of Shu-Di-Huang demonstrate that traditional processing methods can sometimes be of questionable value. Traditionally, nine cycles of "steam-heating and drying” process would be carried out turning the color to be as black as lacquer to obtain "the best quality" of Shu-Di-Huang according to traditional descriptions in the ancient Compendium Materia Medica. However there was no standardized procedure to regulate the operation and no real criteria for evaluation in spite of the request of the root tuber's color. The results of chromatographic fingerprint analysis confirmed that the uncertainty of the total steam-heating procedure makes the quality of the final product inconsistent, even some over-steamed Shu-Di-Huang caused a loss of almost all bioactive components and only fructose remained. It is appealed to the manufacturer the significance for defining the appropriate standard for both Sheng-Di-Huang and Shu-Di-Huang. Referring to the pattern of chromatographic fingerprints, the iridoids should be preserved as more as possible in "Sheng-Di-Huang", and the oligosaccharides, stachyose in particular, should be kept as consistent as possible in "Shu-Di-Huang", the increment of 5-HMF caused by dehydration of glucose in processing period should also be measured as it has its bioactive contribution. 


\section{Acknowledgements}

The corresponding author thanks Professor Du Hengqing for her help in obtaining authentic samples of Di-Huang.

\section{References}

[1] Xiao. P.G. (2002) Modern Chinese Materia Medica, Vol. 2, Chemical Industry Press, Beijing, 390.

[2] The Commission of the PPRC (2005) Pharmacopoeia of People's Republic of China, Vol. 1, Chinese Medicinal Science and Technology Press, Beijing, 115-117.

[3] Tomoda, M., Kato, S. and Onuma M. (1971) Water Soluble Constituents of Rhemanniae Radix I. Carbohydrates and Acids of Rehmannia glutinosa f. hueichingensis. Chemical \& Pharmaceutical Bulletin (Tokyo), 19, 1455-1460. http://dx.doi.org/10.1248/cpb.19.1455

[4] Tomoda, M., Tanaka, M. and Kondo, N. (1971) Water Soluble Constituents of Rehmanniae Radix II: On the Constituents of Roots of Rehmannia glutinosa var. purpurea. Chemical \& Pharmaceutical Bulletin, 19, 2411-2413. http://dx.doi.org/10.1248/cpb.19.2411

[5] Oshio, H. and Inouye, H. (2008) Iridoid Glycosides of Rehmannia glutinosa. Phytochemistry, 21, 133-138. http://dx.doi.org/10.1016/0031-9422(82)80029-0

[6] Tang, W.C. and Eisenbrand, G. (1992) Chinese Drugs of Plant Origin, Subtitle: Chemistry, Pharmacology and Use in Traditional and Modern Medicine, Springer Berlin Heidelberg, 849-854. http://dx.doi.org/10.1007/978-3-642-73739-8_106

[7] Liu, C.H., Zhang, L.J. and Li, G.S. (2002) Determination of Rehmannioside A in Root Tuber of Rehmannia glutinosa. Chinese Traditional and Herbal Drugs, 33, 706-707.

[8] Zhu, M.F., Liu, X.Q., Ju-hee, O., Chang-soo, Y. and Je-Hyun, L. (2007) Effect of Concentration of Catalpol and 5-Hydroxymethyl-2-Furaldehyde from Processing of Rehmanniae Radix. Zhongguo Zhong Yao Za Zhi, 32, 1155-1157.

[9] Zhang, H.-F. (2009) Survey on the Influence of Different Processing Methods to the Contents of Saccharids and Catalpol (in Chinese). Journal of Practical Traditional Chinese Medicine (Zhongguo Shiyan Zhongyao Zazhi), 25, 501-502.

[10] Li, G.S., Liu, C.H. and Wang, H.S. (2002) Comparison of the Content of Catalpol of Dihuang in Different Habitats. Zhong Cao Yao, 33, 12.

[11] Zhang, K., Guo, J.H., Tian, C.W. and Zhang, T.J., (2013) Effects of Various Treatments and Influencing Factors on Catalpol in Rehmannia glutinosa. Chinese Traditional and Herbal Drugs, 44, 896-899.

[12] Chang, W.-T., Choi, Y.H., Van Der Heuden, R., Lee, M.-S., Lin, M.-K., Kong, H.W., Kim, H.K., Verpoorte, R., Hankemeier, T., Van Der Greef, J. and Wang, M. (2011) Traditional Processing Strongly Affects Metabolite Composition by Hydrolysis in Rehmannia glutinosa Roots. Chemical and Pharmaceutical Bulletin, 59, 546-552. http://dx.doi.org/10.1248/cpb.59.546

[13] Wen, X.-S., Yang, S.-L., Ma, X.-J. and Zheng J.-H. (2004) HPLC Chromatogram Changes with Processing for Roots of Radix Rehmanniae. Chinese Traditional and Herbal Drugs (Zhong Cao Yao), 35, 153-154.

[14] Lai, P.K.-K., To, M.-H., Lau, K.-M., Liu, C.-L., Cheng, L., Fung, K.-P., Leung, P.-C. and Lau, C.B.-S. (2012) Stachyose: One of the Active Fibroblast-Proliferating Components in the Root of Rehmanniae Radix (Dihuáng). Journal of Traditional and Complementary Medicine, 2, 227-234

[15] Li, D.Q., Duan, Y.L., Bao, Y.M., Liu, C.P., Liu, Y. and An, L.J. (2004) Neuroprotection of Catalpol in Transient Global Ischemia in Gerbils. Neuroscience Research, 50, 169-177. http://dx.doi.org/10.1016/j.neures.2004.06.009

[16] Li, D.Q., Li, Y., Liu, Y., Bao, Y.M., Hu, B. and An, L.J. (2005) Catalpol Prevents the Loss of CA1 Hippocampal Neurons and Reduces Working Errors in Gerbils after Ischemia-Reperfusion Injury. Toxicon, 46, 845-851. http://dx.doi.org/10.1016/j.toxicon.2004.09.007

[17] Tian, Y.Y., An, L.J., Jiang, L., Duan, Y.L., Chen, J. and Jiang, B. (2006) Catalpol Protects Dopaminergic Neurons from LPS-Induced Neurotoxicity in Mesencephalic Neuron Glia Cultures. Life Sciences, 80, 193-199. http://dx.doi.org/10.1016/j.lfs.2006.09.010

[18] Jiang, B., Liu, J.H., Bao, Y.M. and An, L.J., (2004) Catalpol Inhibits Apoptosis in Hydrogen Peroxide-Induced PC12 Cells by Preventing Cytochrome $c$ Release and Inactivating of Caspase Cascade. Toxicon, 43, 53-59. http://dx.doi.org/10.1016/j.toxicon.2003.10.017

[19] Yu, Z., Wang, J., Li, G.S. and Wang, Y.S. (2001) Experimental Study on Rehmannioside D in the Action of Nourishing Yin, Enriching the Blood and Reducing the Blood Sugar. Liaoning Journal of Traditional Chinese Medicine (Liaoning Zhongyi Zazhi), 28, 240-242.

[20] Guo, X.N., Zhang, R.X., Jia, Z.P., Li, M.X. and Wang, J. (2006) Effects of Rehmannia glutinosa Oligosaccharides on 
Proliferation of 3T3-L1 Adipocytes and Insulin Resistance. Chinese Journal of Chinese Materia Medica (Zhongguo Zhongyao Zazhi), 31, 403-407.

[21] Ding X., Wang, Mi.-Y., Yu, Z.-L., Hu, W. and Cai, B.-C. (2008) Stuides on Separation, Appraisal and the Biological Activity of 5-HMF in Cornusofficinalis. Chinese Journal of Chinese Materia Medica (Zhongguo Zhongyao Zazhi), 33, 392-396. 